Editorial

\title{
Operational research models and methods in the energy sector
}

In September 1957, The Economist reported about the first International Conference on Operational Research, which had been held in Oxford (UK), emphasizing that the majority of (British) papers presented therein were practical case studies. The Economist used as showcase the problem faced by the Central Electricity Authority of calculating how - from which pits to which power stations, in what kinds of transport and over which routes - it could supply the stations (...) with the coal they needed at the minimum cost. A linear programming model achieved economies of $£ 0.3$ million a year, and The Economist concluded that the Central Electricity Authority management had been encouraged enough with the results to widen the exercise towards national scope.

Also, the oil industry has been a heavy user of OR techniques to support refinery operations management and electricity companies rely on OR to schedule generator operations and trade power.

The application of the models and methods of OR has revealed a very effective contribution to the successful resolution and sound decision support in several problems arising in the energy sector since the early days of OR.

The changes underway in the organization of the energy sector, namely in the electric sector with the unbundling of previously vertically integrated activities, the trend towards the liberalization of the energy markets, the environmental problems associated with activities from extraction to end-use of energy, the socio-economic concerns in the framework of sustainable development, bring up new challenges as well as new opportunities for OR.

The energy sector is characterized by a diversity of problems of distinct nature (technical and operational, policy design, short- and long-term planning, systems management, market modelling, etc.), and different players involved (utilities, energy service companies in different activity branches, customers, system and market operators, regulatory bodies, governments, etc.). The OR toolbox offers researchers and practitioners a large and rich set of models and methods to deal with these challenges and problems in a creative and effective manner.

This special issue comprises ten papers presenting the application of models and methods of OR in the energy sector. Techniques used in these contributions include (mixed integer) linear programming, fuzzy models, game theory, stochastic programming, data envelopment analysis and multi-criteria analysis. Areas of application involve the petroleum, gas and electricity industries as well as local and national planning. A vast array of problems is addressed, from technical and operational issues to policy design, planning and management of energy systems. We hope this special issue can be useful for a large audience, both researchers and decision-makers, conveying a value-added to help them tackle the challenging problems to be faced in the energy sector.

A characteristic of most industries, such as the petroleum industry, is the prevalence of production processes leading to joint production costs, whenever the production of a product simultaneously and necessarily involves the production of one or more other products. The linear programming approach has been commonly proposed for joint cost allocation purposes, based on marginal analysis. However, in presence of capacity, institutional or environmental constraints, the additivity property that is required to completely allocate joint costs fails. Tehrani and Michelot propose a two-stage procedure based on the marginal costs and the production elasticity of input factors to achieve an additive cost allocation pattern of the total variable cost of a joint production firm on its joint products. The additive cost allocation is derived exploiting just the information obtained from the optimal simplex tableau. Illustrative results are provided for a refinery case study.

The changes currently underway in the organization of the electric sector, towards the unbundling of the generation, transmission, distribution and commercialization activities, lead to the need of evaluating the adequacy of the transmission system independently of the generation system. Fuzzy approaches seem more adequate than probabilistic models to tackle uncertainty regarding generation due to the market influence concerning scheduled units and generation amounts, and due to their capability to describe mathematically qualitative declarations about load or generation. The power flow computation is an important tool for planning and operating electric power systems. The paper by Gouveia and Matos shows how to perform the detailed calculation of the state variables of the fuzzy power flow problem in an exact and symmetrical way, by means of solving multiple constrained non-linear programming problems in which power flow constraints or voltage constraints can be included.

High-pressure natural gas transportation and distribution systems are very complex structures made up of several pipe sections of different diameters that must be adapted to different conditions of flow and pressure. Gas transportation companies need to plan the reinforcement of those networks. The paper by Andre, Bonnans and Cornibert presents heuristics for solving the large-scale integer non-linear problem of minimizing investment costs of an existing gas transportation network, finding the pipeline segments to be reinforced and their sizes under demand satisfaction constraints with respect to different pressures. The methodological approach consists in solving a continuous relaxation followed by a branchand-bound scheme. 
While liberalizing regulatory orders have sought to provide a more competitive environment, certain market players may now have the ability to exert market power. At the same time there is a growing concern in the US and the EU over supply security due to the reliance on foreign fuel sources. The paper by Gabriel, Zhuang and Egging presents new heuristics (scenario-reduction approaches that iteratively work towards solving the extensiveform stochastic market equilibrium model) to solve stochastic complementarity problems. These methods embody game theoretic and stochastic elements to address the market power potential as well as uncertainty that key players face. These models' computational complexity stems from the fact that players can exert both strategic as well as hedging behaviour.

The deregulation of the energy markets increased the importance of electricity market derivatives such as futures, swaps, caps, floors, collars and options. Electricity swing options are supply contracts for power, which give the owner the right to change the required delivery on short time notice, thus offering more flexibility than fixed base load or peak load contracts. Pflug and Broussev present a method to determine a rational ask price for swing options, which is based on the notion of the least acceptable price for the option seller and a behavioural model for the option holder. A game-theoretic model for identifying the equilibrium price is developed. The pricing method is based on dynamic stochastic optimization.

Pool-type auctions are a group of possible spot market designs, which are well-suited coordination mechanisms for short-term trading on the centralized electricity markets. The pool scheduling problem (day-ahead multi-period auction) can be formulated as a unit commitment optimization problem under competition, balancing production offers (sell bids) with inelastic (or elastic) demand. It has been recognized that marginal pricing of energy is not sufficient to assure efficient self-committed schedules and to support equilibrium allocations in the auction-based markets. Toczylowski and Zoltowska propose a new pricing scheme for determining the social welfare distribution in a centralized pool-based auction in the context of solving the unit commitment problems under competition, including the price-responsive demand side for the multi-period auctions with dynamic commitment characteristics. The model allows every thermal unit and every consumer to obtain individual maximum profits, and offers the market coordinator a tool for solving the technologically constrained unit commitment problem with fair market clearing. The pricing model is a mixed linear programming model aimed at minimizing the sum of the compensation costs.

Strategic decision-making regarding sustainable development embodies challenges arising from the multi-dimensionality of the sustainability goal, from the complexity of socio-economic and biophysical systems, and from the long-term nature of the problems and the associated uncertainties. The paper by Kowalski, Stagl, Madlener and Omann addresses the merits and challenges of combining participatory multi-criteria analysis with scenario building for analysing and aiding decision-making in the context of renewable energy use for promoting sustainable development. Renewable energy scenarios for Austria have been appraised against sustainability criteria both at national and local level. The authors report that combining scenarios and participatory multicriteria analysis contributes to capture the context of technology deployment and allows decision-making based on a robust and democratic process, which addresses uncertainties, acknowledges multiple legitimate perspectives and encourages social learning.

In Multi-Attribute Utility Theory (MAUT) it has been recognized that decision makers may consider as somewhat vague the concept of expected utility values. Løken, Botterud and Holen introduce the idea of the Equivalent Attribute Technique to improve the comprehensibility of a MAUT study by converting a change in the expected total utility into an equivalent quantity in one of the decision attributes, which is often an economic attribute. A simplified linear model and a more advanced non-linear model are considered, this latter including the decision maker's strength of preference and risk attitude. The technique is applied to a local energy planning problem characterized by multiple energy sources and carriers.

The paper by Madlener, Antunes and Dias uses Data Envelopment Analysis (DEA) and Multi-Criteria Decision Analysis (MCDA) for gaining insights about the possibilities of combining these techniques as complementary tools for evaluation purposes. The idea is determining how MCDA methods may be of help in efficiency evaluation, under the same principles of DEA but using MCDA's capabilities of explicitly incorporating managerial preference information. To keep the DEA spirit, the evaluation criteria are defined as output/input efficiency ratios and no information about their weights is introduced at the outset. The approach is illustrated in a case study dealing with the efficiency assessment of agricultural biogas plants in Austria.

The handling of undesirable outputs, which are jointly produced with the desirable outputs, and the treatment of uncontrollable variables, which often capture the impact of the operating environment, are difficulties arising in doing an objective evaluation of the performance of decision-making units (DMUs). DMUs must be credited/penalized for their provision of desirable/undesirable outputs. Also, if the impact of uncontrollable variables is not filtered out, the evaluation of those DMUs in an adverse operating environment will be underestimated by implicitly assuming that all DMUs' inefficiencies are caused by poor management. The paper by Yang and Pollitt proposes six DEA performance evaluation models based on a sample of Chinese coal-fired power plants, considering undesirable outputs (pollutant emissions) and uncontrollable variables.

Carlos Henggeler Antunes Department of Electrical Engineering and Computers Polo II, University of Coimbra, 3030 Coimbra, Portugal E-mail address: ch@deec.uc.pt

Álvaro Gomes

RE'D Unit INESC Coimbra, Rua Antero de Quental 199, 3000-033 Coimbra, Portugal E-mail address: agomes@deec.uc.pt

Available online $\mathrm{xxxx}$ 